The International Journal Of Engineering And Science (IJES)

|| Volume || 5 || Issue || 11 || Pages || PP 34-37 || 2016 ||

ISSN (e): $2319-1813$ ISSN (p): $2319-1805$

THE IJES

\title{
Analysis of the skill of a world-class alpine ski racer by using a 3D CAD system
}

\author{
Soichiro Suzuki ${ }^{1, \mathrm{a}}$, Daichi Taguchi, ${ }^{2, \mathrm{~b}}$, Yohei Hoshino ${ }^{1, \mathrm{c}}$, Cao Ying ${ }^{1, \mathrm{~d}}$ and \\ Lianlian Yang ${ }^{1, \mathrm{e}}$ \\ ${ }^{I}$ Department of Mechanical Engineering, Kitami Institute of Technology, \\ 165 Koen-cho, Kitami, Hokkaido, 090-8507 Japan \\ ${ }^{2}$ Graduate School of Engineering, Kitami Institute of Technology, 165 Koen-cho, Kitami, Hokkaido, 090-8507 \\ Japan
}

\begin{abstract}
Heavy snow areas account for about 50\% of the total land area of Japan. Extension of healthy life expectancy is an important issue for residents of heavy snow areas because most of these areas in Japan are depopulated areas. Skiing is a possible lifelong sport in heavy snow areas because gravity rather than muscular power is mainly utilized in skiing. However, in Japan, the number of skiers has declined to $30 \%$ of the peak number. This study was conducted with the aim of recovery of the popularity of skiing, and establishing skiing as lifelong winter sport. World-level success of Japanese alpine ski racers is an important factor for the recovery of the popularity of skiing. In this study, the skill of an alpine ski racer was investigated by using a 3D CAD model of a skier with focus on joint work and energy balance in the turn motion.
\end{abstract}

Keywords: Alpine ski racing, Skill analysis, Energy balance, Bending motion, Stretching motion, 3D CAD

Date of Submission: 17 May 2016

$\longrightarrow$ Date of Accepted: 12 November 2016

\section{INTRODUCTION}

In Japan, the aging rate, which is the ratio of the population aged 65 years and older to the total population is more than $25 \%$, which is higher than that of any other country in the world. Therefore, extension of healthy life expectancy is an important issue in Japan. Making lifelong sports more popular would be one effective way to extend the healthy life expectancy. Skiing is popular winter sports in many countries, and elderly people can also do skiing because gravity rather than muscular power is mainly utilized. However, in Japan, the number of skiers has recently declined to $30 \%$ of the peak number in the bubble economy period. As a result, $37 \%$ of the ski fields in Japan have been closed [1], and 56\% of the closed ski fields are small-scale ski fields and are managed by the local government of Japan [1]. Therefore, most elderly people who live in cold regions cannot easily do skiing.

This study was carried out with the aim of recovery of the popularity of skiing and establishing skiing as a lifelong winter sport in Japan. The world-level success of Japanese alpine ski racers is an important factor for the recovery of popularity of skiing. However, no Japanese ski racer has won an alpine ski race at the Olympics or world championships. The turning technique of the world's top skiers in alpine ski races might therefore be quite different from that of Japanese skiers. In this study, the skill of an alpine ski racer was examined by focusing on energy balance in the turn motion. Many researches have examined the physical characteristics of alpine ski racers or kinematics of the skier [2-5] and the optimum trajectory of skiing in an alpine ski race [6,7]. However, the kinetics of the skill of an alpine ski racer has not been examined because it is experimentally difficult to extract the characteristics of the skill of the world's top level turning technique in a varied and changeable downhill course. Therefore, in this study, the skill of an alpine ski racer based on energy balance of the skier was analyzed by using 3D CAD in order to extract the characteristics of a skillful turning technique from only broadcasted images of the race without conducting an experiment. 


\section{COMPONENTS OF TURN MOTION}

Fig. 1 shows components of the turn motion of a skier. The turn motion is composed of three parts including two distinctive positions. The first half of the turn motion in which a skier increases the lean angle of his/her lower limb is called the downhill turn. The latter half of the turn motion in which a skier decreases the lean angle is called the uphill turn. Between these two parts, there is a switching zone of the turn direction that includes a neutral position when skis become flat against the slope. At the final stage of the downhill turn, a skier takes a maximum position when the lean angle of the lower leg becomes maximum. As shown in Fig. 2(a) and (b), the motion of a skier at the start of switching the turn direction is classified into two patterns according to knee and hip joint movements. One is called bending motion in which a skier bends his/her knee and hip joints after the maximum position, and the other is called stretching motion.

\section{ANALYSIS OF SKILL BASED ON JOINT WORK}

\subsection{Energy generation}

In alpine ski races, the racers glide down the course by utilizing potential energy caused by the difference between the altitude values of the start and finish points and simultaneously transforming this energy into kinetic energy. Therefore, it is expected that the winner has generated the most kinetic energy by joint work with the least dissipation energy caused by negative work of cutting resistance of snow by the ski edge, air resistance of the skier, friction between the ski sole and snow and viscous resistance of the joints of the skier.

\subsection{Joint work}

The so called bending motion, which is the knee and hip joint movements of a skier at the start of switching the turn direction, may consume energy because viscous resistance occurs during bending of the joints. Therefore, it is expected that there is close relationship between the finishing time of the race and the number of bending motions of the skier from the start to finish. Fig. 3(a) and (b) show the relationship between the number of bending motions and the official record of finishing time for 10 racers in World Cup slalom races held in Kranjska Gora and Wengen, respectively. These 10 racers ranked top $30_{\text {th }}$ in the World Cup and did not make a large mistake in the races. A very strong correlation was found between the number of bending motions and the official record of finishing time, suggesting that only the number of bending motions determines victory among high-ranking World Cup racers and that there is no difference in the energy consumption of one bending motion, cutting resistance of the snow, air resistance or friction of the ski sole among the world's top-level skiers. It was clarified that one bending motion caused a loss of over $0.1[\mathrm{~s}]$ in finishing time judging from the inclination of a linear approximation of the plot in Fig. 3. Consequently, execution of less bending motions and more stretching motions in the race is the most important skill for alpine ski World Cup racers.

\section{FINISHING TIME ESTIMATION BASED ON ENERGY BALANCE}

\subsection{Formulation of energy balance during the downhill descent}

It was demonstrated that the number of bending motions from the start to finish strongly affected the finishing time of high-ranking World Cup racers with no large mistake in the race. This means that decrease of the energy consumption caused by the bending motion and increase of joint work by the stretching motion are the most important factors to shorten the finishing time for top-level World Cup racers. The energy balance of a racer during the downhill descent is expressed as

$$
\begin{aligned}
m v^{2} / 2= & m g h-(\text { C.Loss }) \times N_{T} \\
& -\int_{L_{1}}^{L_{2}}(\text { A.Loss }) d s-(\text { B.Loss }) \times N_{B} \\
& +W \times N_{S},
\end{aligned}
$$

where $m[\mathrm{~kg}]$ is weight of the skier, $v[\mathrm{~m} / \mathrm{s}]$ is downhill velocity and $h[\mathrm{~m}]$ is altitude difference between the start point $L_{1}$ and present position of the skier $L_{2}$. A.Loss [J], B.Loss [J] and C.Loss [J] are dissipation energy caused by air resistance, bending motion and negative work of cutting resistance of the snow in one turn, respectively. $W_{j}[\mathrm{~J}]$ is work of the knee joint and hip joint, $N_{r}$ is the total number of turns from the start to the present position, which is equal to the sum of the number of bending motions $N_{b}$ and the number of stretching motions $N_{s}$.

Assuming that there is no great difference in A.Loss and C.Loss among high-ranking World Cup racers, the difference in kinetic energy among the racers $\Delta m v^{2} / 2[\mathrm{~J}]$ is

$$
\Delta\left(m v^{2} / 2\right)=W \times \Delta N_{S}-(\text { B.Loss }) \times \Delta N_{B},
$$

where $\Delta N_{b}$ and $\Delta N_{s}$ are the differences in the number of bending motions and the number of stretching motions among the racers, respectively. 


\subsection{Estimation of viscoelasticity of the joints}

Estimation of the finishing time using Eq. 2 verifies the skill of lower limb movement of a top-level alpine ski racer. In this estimation, it is necessary to determine the change in joint angles and viscoelasticity of the joints. In order to identify these characteristics, jumping motions of three ski racers wearing ski boots were recorded. When the changes in joint angles at the moment of jumping up and landing and also the height of the jumping motion are measured, viscoelasticity can be estimated by using the statistical mass distribution of the subject. The jumping motions of three top-level Japanese ski racers were recorded. Table 1 and Table 2 show the average values of changes in joint angles and viscoelasticity measured three times in the experiment, respectively.

\subsection{Finishing time estimation}

Table 3: shows the dissipation energy and work that were calculated by estimated viscoelasticity of the joints.

\begin{tabular}{|l|l|c|}
\hline & $\begin{array}{l}\text { Dissipation energy in } \\
\text { bending motion [J] }\end{array}$ & $\begin{array}{l}\text { Joint work in stretching } \\
\text { motion }[\mathrm{J}]\end{array}$ \\
\hline Hip & 4.68 & 5.19 \\
\hline Knee & 8.95 & 10.55 \\
\hline Total & 13.63 & 15.74 \\
\hline
\end{tabular}

\begin{tabular}{|l|l|l|l|}
\hline$c_{1[\mathrm{~N} \cdot \mathrm{m} \cdot \mathrm{s} / \mathrm{rad}]}$ & $c_{2[\mathrm{~N} \cdot \mathrm{m} \cdot \mathrm{s} / \mathrm{rad}]}$ & $k_{1[\mathrm{~N} \cdot \mathrm{m} / \mathrm{rad}]}$ & $k_{2[\mathrm{~N} \cdot \mathrm{m} / \mathrm{rad}]}$ \\
\hline 35.0 & 57.6 & 249.3 & 613.7 \\
\hline
\end{tabular}

\begin{tabular}{|l|l|l|l|}
\hline \multicolumn{2}{|l|}{ Bending [deg] } & \multicolumn{2}{l|}{ Stretching [deg] } \\
\hline Hip & Knee & Hip & Knee \\
\hline-7.87 & -9.26 & 10.97 & 10.79 \\
\hline
\end{tabular}

By inserting these values for B.Loss and $W_{j}$ into Eq. 2, the difference in average values of downhill velocity $\Delta v[\mathrm{~m} / \mathrm{s}]$ is calculated. Consequently, the finishing time of each racer can be estimated by Eq. 3 on the basis of the fastest official finishing time of the winner.

$$
\Delta t=\frac{t_{1}^{2} \cdot \Delta v}{L}
$$

where $\Delta t[\mathrm{~s}]$ is the difference in finishing time from the winner, $t_{1}[\mathrm{~s}]$ is the official finish time of the winner, and $L[\mathrm{~m}]$ is the distance of the downhill course of the race that is calculated by the finishing time and $\Delta v$. Fig. 4 shows a comparison of the finishing time according to the official record and the estimated value in the same race as shown in Fig. 3(a). It was demonstrated that the finishing time was accurately estimated and that energy balance of the joint movement of the lower limb is important to shorten the finish time.

\subsection{Motion capture by a 3D CAD model}

\section{ACCURATE ESTIMATION OF FINISHING TIME}

It was clarified that the finishing time of the world's top-level racers could be accurately estimated by the energy balance of lower limb movement. However, if there is great difference in joint movement among the racers, the estimation time will have a large error. If joint movements of the racers in the same turn are different or the racers have different levels of skill, accurate measurement of joint movement will be required for the estimation. However, it is difficult to invite top-level skiers as experimental subjects to analyze their skill. Therefore, a method that can capture joint movement from broadcasted images of an alpine ski race is needed. At the beginning of this investigation, a 3D CAD model of a skier based on the statistical mass distribution of a young athlete's body
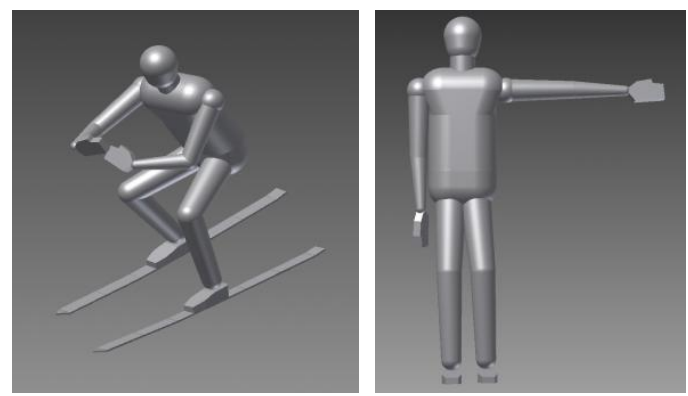

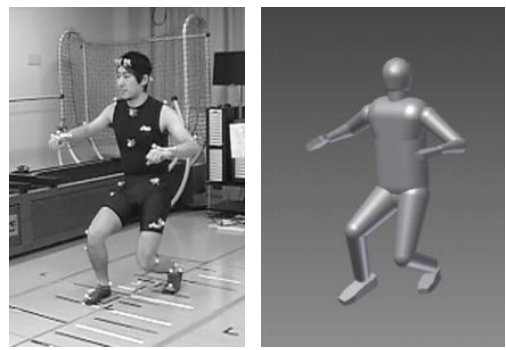

was constructed as shown in Fig. 5. For capturing joint movement from an image, expansion or reduction, 3D rotation and changing the joint angle of the CAD model by manual adjustment are required. The accuracy of this motion capture method was compared with that of the VICON 460 system as shown in Fig. 6 (a) and (b). The average error of joint movement captured by 3D CAD was under $0.5 \%$ as shown in Table 4 . It was clarified that the motion capture method using 3D CAD was sufficiently accurate to analyze the skill of a skier.

\subsection{Estimation of finishing time using the 3D CAD model}

Work of the stretching motion and dissipation energy of the bending motion in every turn were calculated for each racer using joint movement captured by the 3D CAD model. Fig.7 shows the results of estimation of finishing time in the 2014 Levi Slalom World Cup based on the assumption that all racers have the same joint movement. Fig. 8 shows the results of estimation by using the 3D CAD model. A comparison of the results showed that finishing time could be accurately estimated by the motion capture method using the 3D CAD model.

\begin{tabular}{|l|l|l|l|l|}
\hline \multirow{2}{*}{$\begin{array}{l}\text { Trial } \\
\text { No. }\end{array}$} & \multicolumn{2}{|l|}{ Error [deg] } & $\begin{array}{l}\text { Ratio for the angle change } \\
{[\%]}\end{array}$ \\
\cline { 2 - 5 } & Average & Maximum & Average & Maximum \\
\hline 1 & 0.2 & 0.3 & 0.3 & 0.5 \\
\hline 2 & 0.2 & 0.5 & 0.3 & 0.6 \\
\hline 3 & 0.3 & 0.6 & 0.5 & 0.8 \\
\hline
\end{tabular}

The results suggested that the motion capture method is useful for analyzing the skill of a top-level skier.

\section{SUMMARY}

In this study, the skill of a World Cup alpine ski racer was investigated by using a 3D CAD model of a skier with focus on joint work and energy balance in the turn motion with the aim of recovery of the popularity of skiing as a leisure sport and the establishing of skiing as a lifelong winter sport in Japan. It was demonstrated that there was a very strong correlation between finishing time of the race and number of bending motions at the time of switching the direction of the turn. In addition, work of the hip joint and knee joint was calculated by using a 3D CAD model of the skier matched to the scene of the turn from the broadcasted images of World Cup alpine ski races. It was clarified that the finishing time of each ski racer could be accurately estimated by energy balance of the joint work even if ski racers had different joint movements during the turn motion. The results suggest that the proposed motion capture method is useful for analyzing the skill of a toplevel skier without conductiong an experiment on the snow.

\section{ACKNOWLEDGMENTS}

This work was supported by JSPS KAKENHI (Multi-year Fund) Grant Number (26420191).

Reference an article:

\section{REFERENCES}

[1]. M. Kureha, Regional pattern of lost and closed ski fields in Japan, J. Ski Science. Vol.11 No.1 (2014) 43-50.

[2]. T. Sato, K. Sakuraba, Y. Tsuchiya, S. Maruyama, E. Ochi, Seasonal variation of bone metabolism and bone mineral density in male alpine skiers, J. Ski Science. Vol.11 No.1 (2014) 43-50.

[3]. Y. Kondo, T. Takeda, J. Kawaguchi, Structure of qualitative development of techniques and division of the skill level in giant slalom of alpine skiing competition, J. Ski Science. Vol.9 No.1 (2012) 1-23.

[4]. G. Neumayr, H. Hoertnagl, R.Pfister, A. Koller, G.Eibl, E. Raas, Physical and Physiological Factors Associated with Success in Professional Alpine Skiing, Int

[5]. J. Sports Med. 24 (2003) 571-575.

[6]. E. M. Haymes, A. L. Dickinson, Characteristics of elite male and female ski racers, Medicine and science in sports and exercise. 12 (1980) 153-158.

[7]. Y. Hirano, Quickest descent line during alpine ski racing, Sports engineering. 9 (2006) 221-228.

[8]. F. Seifriz, J. Mester, Measurement and computer simulation of trajectories in alpine skiing, Science and skiing II (2000) 155-164.

[9]. A. Koga, S. Suzuki, S. Hayashi, Y. Hoshino, D. Taguchi, Skill analysis of turn technique focused on viscoelasticity of joints in alpine ski race, Proceedings of SOBIM 2014 (2014) 179-182.

[10]. S. Suzuki, S. Hayashi, D. Taguchi, A. Koga, R. Ishibashi, Y. Hoshino, Skill analysis of alpine ski racer focused on viscoelasticity characteristics of joints, Proceedings of MECJ-15 (2015) in CD. 